\title{
Analysis of rare and endemic flora in northeastern Algeria: the case of the wilaya of Souk Ahras
}

\author{
Leïla TouAti $^{1 *}$, Tarek HAMEL ${ }^{2}$, Amel Meddad-HAmZA ${ }^{1}$, Gérard de BÉLAIR ${ }^{3}$ \\ Manuscrit reçu le 12 mars 2021 et accepté le 3 juillet 2021
}

${ }^{1}$ Laboratory of Environmental Biosurveillance, Department of Biology, Faculty of Sciences, Badji Mokhtar University, Annaba 23000, Algeria.

${ }^{2}$ Laboratory of Plant Biology and Environment, Department of Biology, Faculty of Sciences, Badji Mokhtar University, Annaba 23000, Algeria.

${ }^{3}$ Badji Mokhtar University, Annaba 23000, Algeria.

\begin{abstract}
Scarcity and endemism are considered the most important concepts of a region's biodiversity and conservation. Nevertheless, our understanding of the models of scarcity and endemism is limited to high biodiversity regions as, for example, the wilaya of Souk Ahras, northeastern Algeria. In this study, we have compiled a list of all heritage species, their taxonomic composition, and geographic distribution. A total of 119 species was documented, and their distribution was as analyzed in the biological environments of two distinct phytogeographic sectors - $\mathrm{C} 1$ and $\mathrm{H} 2$. The rate of scarcity and endemism increased alongside the organic matter richness and, as a result, the forest and pre-forest area supported an over-representation of these species. The preservation of this biodiversity, exceptional and threatened, urgently requires appropriate scientific studies and environmental protection as short term measures.
\end{abstract}

Keywords: biogeography, conservation, endemism, rarity, Souk Ahras, threat.

\section{Introduction}

The distribution and abundance of species are key in ecology and biogeography (Huang et al., 2016). The concept of endemism states that a taxon is limited in its distribution to a distinct area, which is the heart of biogeography (Anderson, 1994), and an important criterion for the conservation of biodiversity on a global, national, and local scales (Myers et al., 2000; Riemann \& Ezcurra, 2005). Rare plants have great conservation value, either for their heritage or their risk of extinction (Gaston, 1991; Pimm et al., 1998).

\footnotetext{
*Email for corresponding author: 1.touati@univ-soukahras.dz
} 
The identification of the vital areas for preserving biodiversity, with high concentration of closely related species (Myers et al., 2000; Médail and Diadema, 2006), becomes a fundamental task for the biogeography conservation (Cañadas et al., 2014; Morrone, 2018). With a richness of 25000 vascular plant species, half of which are endemic and are well adapted to dry periods (Quézel, 1995; Véla and Benhouhou, 2007), among the 34 hotspots in plant diversity on the planet (Myers, 2003), the Mediterranean basin is ranked as the third richest hotspot (Mittermeier et al., 2004), with additional complex geological, biological and cultural character (Blondel et al., 2010).

Algeria, due to its geographical position, is part of this hotspot with 4449 taxa, $6.5 \%$ of which are endemic (Dobignard and Chatelain, 2010-2013), with a high rarity index. More than three quarters $(77.9 \%)$ of the strict endemic taxa of Algeria are rare plants, representing less than a quarter of the total number of plants (Véla and Benhouhou, 2007). Despite being classified as a biodiversity hotspot, several regions of this country are yet to be explored (Benhouhou et al., 2018).

In order to safeguard the plant biodiversity of these threatened regions, it is imperative to establish benchmarks that describes their biodiversity (Primack et al., 2012), by updating the taxonomic nomenclature of the Algerian flora (Amirouche and Misset, 2009). This can be carried out with collaboration between scientists and amateurs, active field work, and taxonomic problem solving (Domina et al., 2015).

Work on rare and endemic flora in Algeria has been excessively confined to its geography (Hamel et al., 2013; Zedam, 2015; Miara et al., 2017, 2018; Mansouri et al., 2018; Djebbouri and Terras, 2019; Gordo and Hadjadj-Aoul, 2019). Furthermore, the touchstone study on endemic and/or rare plants in Algeria by Véla and Benhouhou (2007) was based on old data from the flora of Quézel and Santa (1962-1963).

It is in this context that the current work is rooted, proposing an inventory and an update on the rare and endemic flora present in the wilaya (or department) of Souk Ahras. Thereby, here we suggest a starting point for new conservation projects and research on local flora.

\section{Materials and Methods}

\subsection{Study area}

The wilaya of Souk Ahras, located in the extreme northeast of Algeria, is limited in the north and west by the wilayas of El Tarf and Guelma, southwest by the wilaya of Oum el Bouaghi, 
southeast by the wilaya of Tebessa, and in the east by Tunisia (Figure. 1). This region is part of the $11^{\text {th }}$ regional biodiversity hotspot in the Mediterranean, called "Kabylias-NumidiaKroumiria" (Véla and Benhouhou, 2007), and covers the Important Plant Area (IPA) called “El Kala 2" (Yahi et al., 2012; Benhouhou et al., 2018).

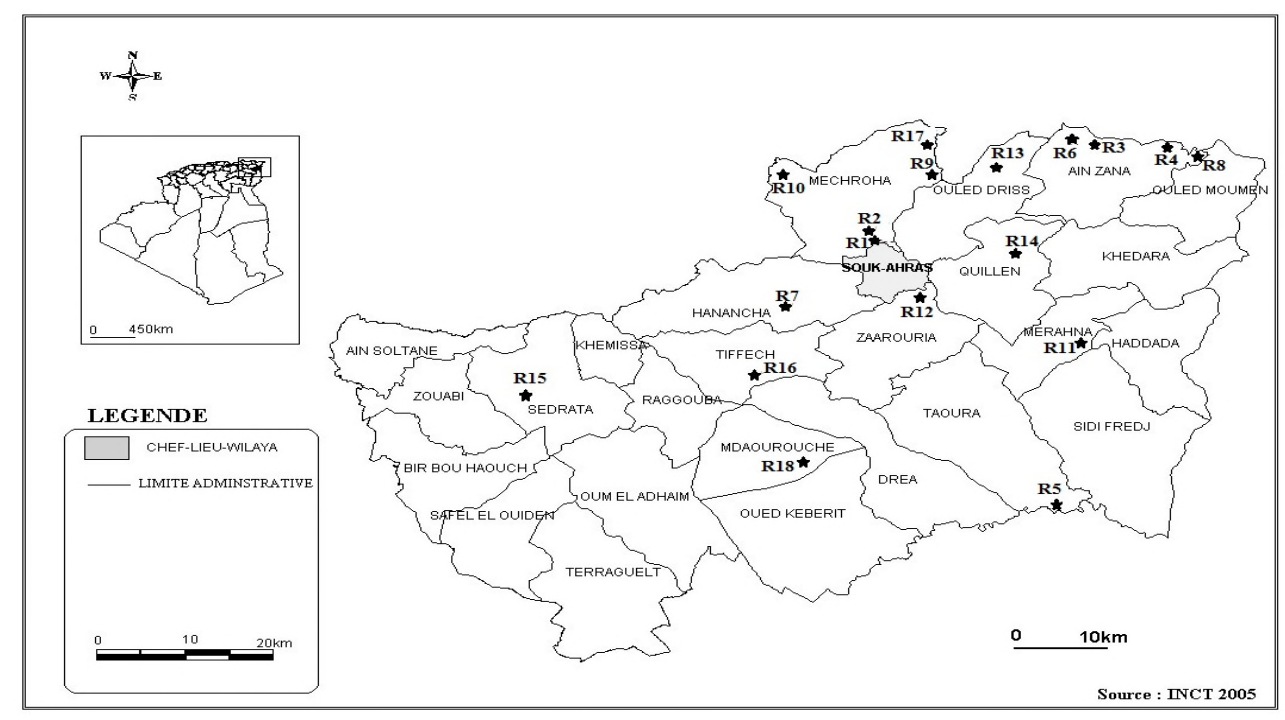

Figure 1: Geographical location of the study sites

Two heterogeneous sets determined the geomorphological configuration of this wilaya: the north $(\mathrm{C} 1)$ was represented by mountains and forests and made up of 12 municipalities, with a total area of $1880 \mathrm{~km}^{2}$, characterized by a humid and subhumid bioclimates, with an average rainfall of $730 \mathrm{~mm} /$ year; the south (H2) made up of high plains and pastures encompassing 14 municipalities, covering an area of $2480 \mathrm{~km}^{2}$, and characterized by a semi-arid bioclimate, with an average rainfall of $350 \mathrm{~mm} /$ year (Hamaidia and Berchi, 2018).

The average daily temperature varied according to the seasons (from $10{ }^{\circ} \mathrm{C}$ in January to $47^{\circ} \mathrm{C}$ in August). Average monthly temperatures were $15^{\circ} \mathrm{C}$ in January and $35^{\circ} \mathrm{C}$ in July (CONM, 1990-2020). In this wilaya, the wooded area amounted to 82,375 ha. It comprised of two definite parts separated by the Medjerda wadi: to the north the cork oak (Quercus suber L.) and zeen oak (Quercus canariensis Willd.), and to the south the Pinus halepensis Mill. (Boudy, 1955). The ecosystems were similar as those of the rest of Numidia (forests, maquis, humid environments, and lawns), except for the coast and the dune compounds (see de Bélair et al., 2005). 
Bulletin de la Société Royale des Sciences de Liège, Vol. : 90, articles, 2021, p. 213 - 240

\subsection{Methodology}

\subsubsection{Floristic study}

The list of rare and endemic species was established according to a systematic sampling, from 2017 to 2020, from floristic surveys. This data collection was carried out at the level of forests of zeen oak (Quercus canariensis Willd.), cork oak (Quercus suber L.), matorrals of Pistacia lentiscus L., Olea europaea L., rocky areas, water bodies, neighboring lawns, and steppes (Macrochloa tenacissima (L.) Kunth and Artemisia herba-alba Asso), according to the phytosociological method of Braun-Blanquet et al. (1952).

At the level of each site, ecological parameters were studied, namely the specific richness of rare and endemic species, altitude, and precipitation (see Table 1).

Table 1: The coordinates of the sampled stations

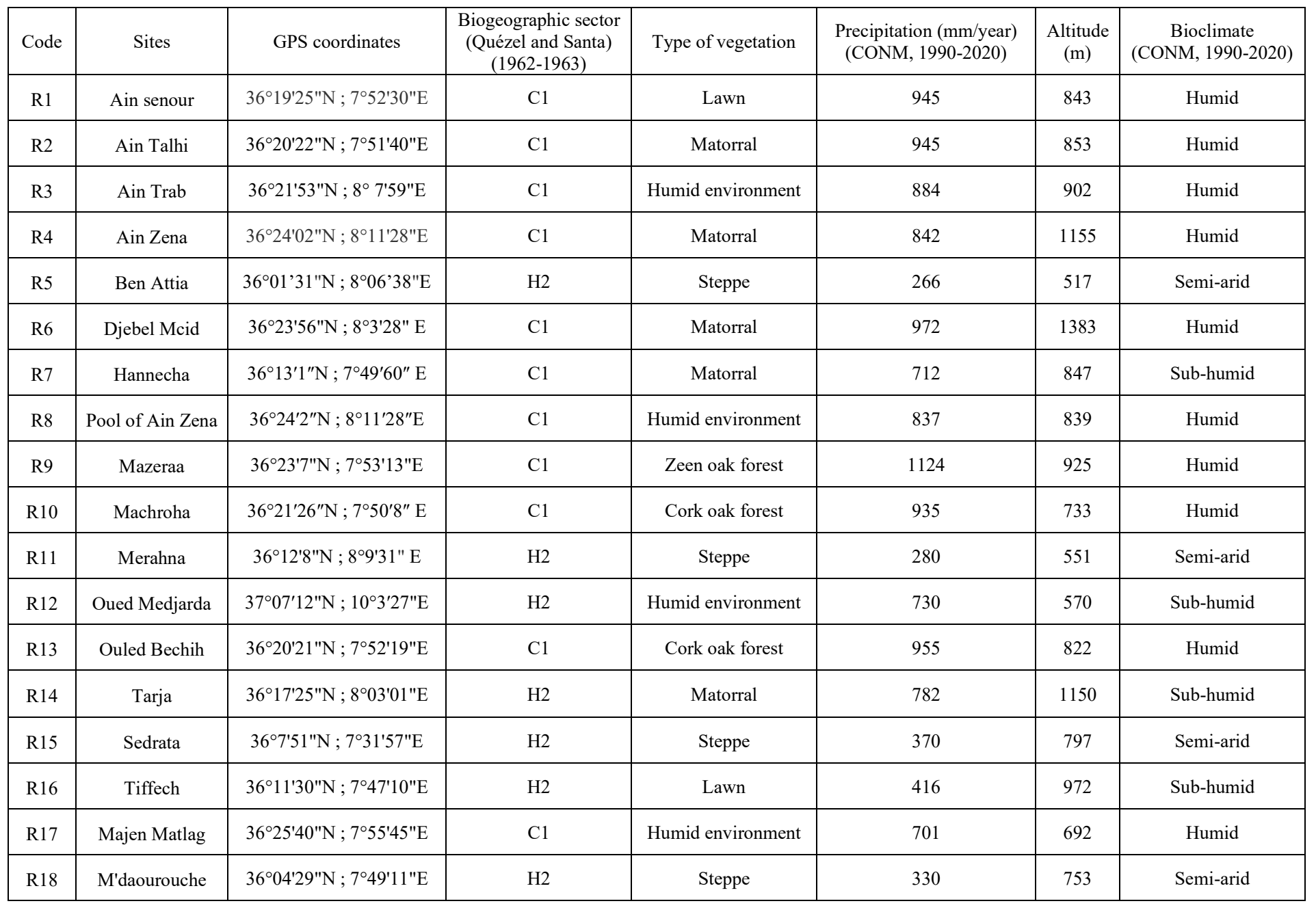


The taxa were identified according to the flora of Battandier (1888-1890), Battandier and Trabut (1895), Maire (1952-1987), Quézel and Santa (1962-1963), Pignatti (1982), and ; Blanca et al. (2009). The nomenclature has been updated according to the synonymic index of North Africa (Dobignard and Chatelain, 2010-2013) and the African Plant Database (APD, 2020). The rarity of taxa was in referrence to the flora of Quézel and Santa (1962-1963) and according to our observations in the field, namely the following statuses: rare $(R)$ and very rare (RR) for non-endemic taxa. For endemic taxa, the classification was as follows: common (C), fairly common (FC), and fairly rare (FR).

The biological types (Raunkiaer, 1934) of the different taxa has been assigned based on Pignatti (1982), Blanca et al. (2009) and, for some endemics, according to Quézel and Santa (1962-1963).

Chorological characterization was carried out based in the flora of Andalusia (Blanca et al., 2009), whereas the eight endemic sub-elements followed the flora of Italia (Pignatti, 1982) and the synonymic index of Dobignard and Chatelain (2010-2013).

\section{Threats and protection status}

The characterization of the on-site threatened species was carried out based on the criteria of rarity by Quézel \& Santa (1962-1963), criteria of vulnerability on a global scale by the International Union for the Conservation of Nature in 1997 (Walter \& Gillett, 1998), and according to the current red list available (IUCN, 2021). The red list makes it possible to highlight the taxa at the highest risk of extinction and defines the priorities in policies to safeguard and conserve the plant biodiversity. We also considered species with heritage interest protected by Decree No. 03-12 / 12-28, in addition to the non-cultivated plant species protected in Algeria (JORA, 2012) and the synonymic index of North Africa (Dobignard and Chatelain, 2010-2013).

\subsection{Scarcity}

Of the 119 species recorded in the study region, nine taxa were quite rare, 55 taxa were rare, 13 taxa were very rare (Table 2), and 14 taxa were rather rare endemics. Indeed, the species observed in the study region seldom had the same heritage value. Additionally, 33 taxa were both endemic and rare (e.g. Bunium crassifolium, Convolvulus durandoi, Dactylorhiza elata, Ophrys subfusca subsp. battandieri). Such relationship between scarcity and endemism was noticeable in our studied flora. Our list includes 28 taxa widely distributed in the national territory (e.g., Anarrhinum pedatum, Stachys duriaei, Bellevalia mauritanica, Rupicapnos 
Bulletin de la Société Royale des Sciences de Liège, Vol. : 90, articles, 2021, p. 213 - 240

numidica), 40 rare to very rare Mediterranean taxa (sensu lato), three rare paleotemperate taxa, and one rare Eurasian taxon.

\begin{tabular}{|c|c|c|c|c|c|c|}
\hline Taxa & Family & $\begin{array}{c}\text { Biological } \\
\text { types }\end{array}$ & Chorological types & Locality & Habitat & Scarcity \\
\hline Achillea ligustica All. & Asteraceae & Hem & Mediterranean & Mazeraa & Zeen oak forest & VR \\
\hline $\begin{array}{l}\text { Allium porrum subsp. } \\
\text { polyanthum (Schult. \& Schult. } \\
\text { f.) Jauzein \& J.-M. Tison }\end{array}$ & Amaryllidaceae & Geo & $\begin{array}{c}\text { Eury- } \\
\text { Mediterranean }\end{array}$ & $\begin{array}{l}\text { Ben Attia, } \\
\text { Tiffech }\end{array}$ & Steppe, lawn & $\mathrm{R}^{*}$ \\
\hline Althaea hirsuta $\mathrm{L}$. & Malvaceae & Th & $\begin{array}{c}\text { Eury- } \\
\text { Mediterranean }\end{array}$ & Ain senour & lawn & $\mathrm{R}$ \\
\hline Ambrosina bassii $\mathrm{L}$. & Araceae & Geo & Subend. Tyrrhenian & $\begin{array}{l}\text { Mazeraa, Ouled } \\
\text { Bechih }\end{array}$ & $\begin{array}{l}\text { Zeen oak forest, } \\
\text { cork oak forest }\end{array}$ & $\mathrm{C}$ \\
\hline $\begin{array}{l}\text { Anacamptis pyramidalis (L.) } \\
\text { Rich. }\end{array}$ & Orchidaceae & Geo & Mediterranean & Ain Talhi & Matorral & $\mathrm{R}^{*}$ \\
\hline Anarrhinum pedatum Desf. & Plantaginaceae & Hem & End. Alg-Tun-Mor & Ain Zena & Cliff & $\mathrm{C}$ \\
\hline $\begin{array}{l}\text { Antirrhinum tortuosum Bo ex } \\
\text { Vent. }\end{array}$ & Plantaginaceae & $\mathrm{Ch}$ & Mediterranean & $\begin{array}{l}\text { Ain Zena, } \\
\text { Hannecha }\end{array}$ & Cliff, matorral & $\mathrm{R}$ \\
\hline $\begin{array}{l}\text { Arabis pubescens (Desf.) Poir. } \\
\text { subsp. pubescens }\end{array}$ & Brassicaceae & Hem & End Alg-Tun-Mor & Ain Zena & Zeen oak forest & FC \\
\hline Arenaria cerastioides Poir. & Caryophyllaceae & Th & End Alg-Tun-Mor & Ain Zena & Zeen oak forest & FC \\
\hline Argyrolobium saharae Pomel & Fabaceae & $\mathrm{Ch}$ & End Alg-Mor-Egy & Ben Attia & Steppe & $\mathrm{R}$ \\
\hline Aristolochia paucinervis Pomel & Aristolochiaceae & Geo & Subend. Tyrrhenian & Ain Trab & $\begin{array}{l}\text { Humid } \\
\text { environment }\end{array}$ & $\mathrm{R}$ \\
\hline Armeria choulettiana Pomel & Plumbaginaceae & Hem & End Alg-Tun-Mor & Ain Talhi & Matorral & FR \\
\hline $\begin{array}{l}\text { Barnardia numidica (Poir.) } \\
\text { Speta }\end{array}$ & Asparagaceae & Geo & End Alg-Tun-Lib & $\begin{array}{c}\text { Mazeraa, Ouled } \\
\text { Bechih, Ain Zena }\end{array}$ & $\begin{array}{l}\text { Zeen oak forest, } \\
\text { cork oak forest, } \\
\text { cliff }\end{array}$ & $\mathrm{C}$ \\
\hline Bellevalia mauritanica Pomel & Asparagaceae & Geo & $\begin{array}{c}\text { End Alg-Tun-Mor- } \\
\text { Lib-Egy }\end{array}$ & Tarja & Matorral & FC \\
\hline Biscutella raphanifolia Poir. & Brassicaceae & Hem & End Alg-Tun-Itl & $\begin{array}{l}\text { Ain Talhi, } \\
\text { Tiffech }\end{array}$ & Matorral, lawn & FR \\
\hline $\begin{array}{l}\text { Brassica procumbens (Poir.) } \\
\text { O.E. Schulz }\end{array}$ & Brassicaceae & Th & End Alg-Tun & $\begin{array}{c}\text { Ain Talhi, Ain } \\
\text { Zena, Ben Attia, } \\
\text { Ouled Bechih, } \\
\text { Mazeraa }\end{array}$ & $\begin{array}{l}\text { Matorral, cliff, } \\
\text { steppe, cork oak } \\
\text { forest, zeen oak } \\
\text { forest }\end{array}$ & $\mathrm{C}$ \\
\hline $\begin{array}{l}\text { Bunium crassifolium (Batt.) } \\
\text { Batt. }\end{array}$ & Apiaceae & Geo & End Alg-Tun & $\begin{array}{l}\text { Machrouha, Ain } \\
\text { Zena }\end{array}$ & $\begin{array}{l}\text { Cork oak forest, } \\
\text { cliff }\end{array}$ & $\mathrm{R}$ \\
\hline Cachrys libanotis L. & Apiaceae & Hem & Mediterranean & Hannecha & Matorral & $\mathrm{R}$ \\
\hline Calamintha menthifolia Host. & Lamiaceae & Hem & Mediterranean & Mazeraa & $\begin{array}{l}\text { Humid } \\
\text { environment }\end{array}$ & $\mathrm{R}$ \\
\hline $\begin{array}{c}\text { Calendula suffruticosa subsp. } \\
\text { boissieri Lanza }\end{array}$ & Asteraceae & Hem & End Alg-Tun-Mor & $\begin{array}{l}\text { Hannecha, Ain } \\
\text { Zena }\end{array}$ & Matorral, cliff & $\mathrm{R}$ \\
\hline $\begin{array}{l}\text { Cardopatium amethystinum } \\
\text { Spach }\end{array}$ & Asteraceae & Hem & End Alg-Tun & Merahna & Steppe & FR \\
\hline Castanea sativa Mill. & Fagaceae & $\mathrm{Ph}$ & Mediterranean & Mazeraa & Zeen oak forest & VR \\
\hline $\begin{array}{l}\text { Cedrus atlantica (Endl.) } \\
\text { Carrière }\end{array}$ & Pinaceae & $\mathrm{Ph}$ & End Alg-Mor & Djebel Mcid & Matorral & $\mathrm{FC}$ \\
\hline Centaurea involucrata Desf. & Asteraceae & Th & End Alg-Mor & Ben Attia & Steppe & $\mathrm{FR}^{*}$ \\
\hline $\begin{array}{c}\text { Centaurea solstitialis } \\
\text { subsp. schouwii (DC.) Gugler }\end{array}$ & Asteraceae & Hem & End Alg-Tun-Itl & Tiffech & Lawn & VR \\
\hline Chaerophyllum temulum $\mathrm{L}$. & Apiaceae & Hem & Eurasian & Majen Matlag & $\begin{array}{l}\text { Humid } \\
\text { environment }\end{array}$ & $\mathrm{R}$ \\
\hline Convolvulus durandoi Pomel & Convolvulaceae & Geo & End Alg-Tun & $\begin{array}{l}\text { Ain Talhi, Ain } \\
\text { zena }\end{array}$ & Matorral, cliff & $\mathrm{R}$ \\
\hline $\begin{array}{l}\text { Cosentinia vellea (Aiton) Tod. } \\
\text { subsp. vellea }\end{array}$ & Sinopteridaceae & Hem & $\begin{array}{c}\text { Eury- } \\
\text { Mediterranean }\end{array}$ & Ain Zena & Cliff & $\mathrm{R}^{*}$ \\
\hline $\begin{array}{c}\text { Cyclamen africanum Boiss. \& } \\
\text { Reut. }\end{array}$ & Primulaceae & Geo & End Alg-Tun-Mor & $\begin{array}{l}\text { Mazeraa, } \\
\text { Mechrouha, } \\
\text { Ouled Bechih, } \\
\text { Ain Zena }\end{array}$ & $\begin{array}{l}\text { Zeen oak forest, } \\
\text { cork oak forest, } \\
\text { cliff }\end{array}$ & $\mathrm{C}$ \\
\hline
\end{tabular}


Bulletin de la Société Royale des Sciences de Liège, Vol. : 90, articles, 2021, p. 213 - 240

Cynosurus polybracteatus Poir.
Dactylorhiza elata (Poir.) Soó
Daucus gracilis Steinh.
Daucus virgatus (Poir.) Maire
Deverra scoparia Coss. \&
Durieu subsp. scoparia
Diatelia tuberaria (L.) Demoly
Drimia anthericoides (Poir.)
Véla \& De Bélair

Drimia numidica (Jord. \&
Fourr.) J.C. Manning \&
Goldblatt

Poaceae

Orchidaceae

Apiaceae

Apiaceae

Apiaceae

Cistaceae

Asparagaceae

Asparagaceae Goldblatt

Ebenus pinnata Aiton
Echinops bovei Boiss.
Eryngium pusillum L.
Euphorbia cuneifolia Guss.
Galactites mutabilis Durieu
Genista ferox (Poir.) Dum.
Cours. subsp. ferox
Genista tricuspidata Desf.
subsp. tricuspidata
Genista ulicina Spach

Fabaceae

Asteraceae

Apiaceae

Euphorbiaceae

Asteraceae

Fabaceae

Fabaceae

Fabaceae

Geranium atlanticum Boiss.

Geraniaceae

Geranium columbinum L.

Geranium dissectum L.

Hedera algeriensis Hibberd

Helosciadium crassipes W.J. Koch

Hertia cheirifolia (L.) Kuntze

Hippocrepis atlantica Ball
Hyacinthoides lingulata (Poir.)
Rothm.

Hypericum androsaemum $\mathrm{L}$.

Hypericum montanum $\mathrm{L}$.

Illecebrum verticillatum $\mathrm{L}$.
Asteraceae

$\mathrm{Ph}$

Geraniaceae

Geraniaceae

Araliaceae

Apiaceae

Fabaceae

Asparagaceae

Hem

Hypericaceae

Hypericaceae

Caryophyllaceae

Th

Th

$\mathrm{Ph}$

Hydr

Geo

$\mathrm{Ch}$

Hem

Th
Th

Geo

Hem

$$
\text { End Alg-Tun }
$$

End Alg-Tun

Th

Hem

Hem

$\mathrm{Ch}$

Geo

End Alg-Tun-Mor

End Alg-Tun

End Alg-Tun-MorLib-Mau

Mediterranean

End Alg

Geo

End Alg-Tun-Spa

End-Alg-Tun-MorLib

Hem

Hem

Th

Th

End Alg-Tun-Mor

Mediterranean

End Alg-Tun-Itl

End Alg-Tun

$\mathrm{Ph}$

End Alg-Tun-Itl

$\mathrm{Ph}$

End Alg-Tun-Mor

$\mathrm{Ph}$

End Alg-Tun

Hem

End Alg-Tun-Mor

Paleotemperate

Paleotemperate

End Alg-Tun

Mediterranean

End Alg-Tun

End Alg-Tun-Mor

Mediterranean

Mediterranean
Mazeraa

Ain Trab

Mechrouha, Ain Zena

Ain senour, Ain

Zena

Ben Attia,

Merahna

Mechrouha

Ain Talhi

Ain Talhi, Ain senour,

Mechroha,

Mazeraa, Mare

Ain Zena, Ain

Zena, Tarja,

Djebel Mcid,

Ouled Bechih,

Ain Trab, Majen Matlag

Tarja, Tiffech, Ben Attia,

Sedrata,

M'daourouche

Ain Talhi, Ain

Zena, Tiffech

Mare Ain Zena,

Majen Matlag

Majen Matlag

Ain senour, Ain

Talhi, Mechroha,

Ain zena, Tarja,

Ouled Bechih, Tiffech

Ain Talhi,

Mechroha, Tarja,

Ain Zena, Ouled

Bechih, Mazeraa

Mechrouha

Ain Talhi,

Mechrouha,

Ouled Bechih,

Ain Zena, Djebel

$$
\text { Mcid }
$$

Djebel Mcid

Majen Matlag

Majen Matlag, Ain Trab

Mazeraa

Mare Ain Zena,

Ain Trab, Majen

Matlag

Ben Attia,

Merahna,

Sedrata,

M'daourouche

$$
\text { Ain Zena }
$$

Mechrouha,

Mazeraa

Mazeraa, Ouled

Bechih

Djebel Mcid,

Ouled Bechih

Mare Ain Zena
Zeen oak forest

Humid

environment

Cork oak forest, cliff

Matorral, cliff

Steppe

Cork oak forest

Matorral

C

$\mathrm{R}$

FR

R

C

$\mathrm{R}$

$\mathrm{R}^{*}$

Matorral, lawn,

Zeen oak forest, cliff, humid

environment, cork oak forest

Matorral, steppe

C

Matorral, cliff,

lawn

Humid

environment

Humid

environment

Matorral, lawn,

cliff, cork oak

forest, Zeen oak forest

Matorral, cork oak forest, cliff, Zeen oak forest

Cork oak forest

C

Matorral, cork oak forest, cliff

FR

Matorral C

Humid environment

Humid

environment

Zeen oak forest

Humid

environment

Steppe

Cliff

R

FR

FR

C

C

$\mathrm{R}$

$\mathrm{R}$

C

VR

C

Cork oak forest, Zeen oak forest Zeen oak forest,

cork oak forest

Matorral, cork oak

forest

Humid

environment
C

(n)

R

(

R


Bulletin de la Société Royale des Sciences de Liège, Vol. : 90, articles, 2021, p. 213 - 240

Iris unguicularis Poir.
Jonopsidium albiflorum Durieu
Juncus heterophyllus Dufour
Lathyrus latifolius subsp.
algericus (Ginzb.) Dobignard
Lepidium rigidum Pomel
Linaria virgata
subsp. algeriensis Murb.
Linum corymbiferum Desf.
subsp. corymbiferum
Linum tenue Desf. subsp. tenue
Lithospermum tenuiflorum L. f.
Lonchophora capiomontana
Durieu
Mandragora officinarum L.
Moricandia suffruticosa (Desf.)
Coss. \& Durieu
Myriophyllum alterniflorum
DC.
Neotinea maculata (Desf.)
Stearn

Oenanthe virgata Poir.

Ononis angustissima subsp. polyclada Murb.

Ononis aragonensis Asso

Ophioglossum lusitanicum L.

Ophrys ×joannae Maire

Ophrys atlantica Munby subsp. atlantica

Ophrys atlantica subsp. hayekii

(H. Fleischm. ex Soó) Soó

Ophrys iricolor Desf. subsp. iricolor

Ophrys marmorata subsp. caesiella (P. Delforge) Véla

Ophrys numida Devillers-

Tersch. \& Devillers

Ophrys subfusca subsp.

battandieri (E.G. Camus) Kreutz

Orchis anthropophora (L.) All.

Orchis laeta Steinh.

Orchis patens Desf. subsp. patens

Origanum vulgare subsp. glandulosum (Desf.) Ietsw.

Orobanche rapum-genistae Thuill.

Phlomis bovei de Noé

Phlomis herba-venti subsp. pungens (Willd.) Maire ex De Filipps

Pilularia minuta Durieu

Pistacia atlantica Desf.

Plagius grandis (L.) Alavi \& Heywood

$$
\text { Iridaceae }
$$

Brassicaceae

Geo

End Alg-Tun

Juncaceae

Fabaceae

Brassicaceae

Plantaginaceae

Linaceae

Linaceae

Boraginaceae

Brassicaceae

Solanaceae

Brassicaceae

Haloragaceae

Orchidaceae

Apiaceae

Fabaceae

Fabaceae

Ophioglossaceae

Orchidaceae

Orchidaceae

Orchidaceae

Orchidaceae

Orchidaceae

Orchidaceae

Orchidaceae

Orchidaceae

Orchidaceae

Orchidaceae

Lamiaceae

Orobanchaceae

Lamiaceae

Lamiaceae

Marsileaceae

Anacardiaceae

Asteraceae
Th

End Alg-Tun-Itl

Hydr

Hem

Hem

$\mathrm{Ch}$

Hem

Th

Ch

Th

Hem

Hem

Hydr

Geo

Hem

Ch

$\mathrm{Ph}$

Geo

Geo

Geo

Geo

Geo

Geo

Geo

Geo

Geo

Geo

Geo

Ch

Geo

Hem

Hem

Hydr

$\mathrm{Ph}$

$\mathrm{Ch}$

Mediterranean

Mediterranean

Mediterranean-

Atlantic

Mediterranean -

Atlantic

Mediterranean

End Alg-Tun

Mediterranean

Mediterranean

Mediterranean

End Alg-Tun

MediterraneanAtlantic

End Alg-Tun

End Alg-Tun-Itl

End Alg-Tun

Eury-

Mediterranean

Mediterranean

Mediterranean

End Alg-Tun

Mechrouha,
Mazeraa
Djebel Mcid,
Tarja
Mare Ain Zena,
Majen Matlag
Ain Talhi
Ain senour
Sedrata

Cork oak forest, zeen oak forest

Mechrouha

Mechrouha

End. Alg-Mar-Spa

End Alg-Tun-Lib

End Alg-Tun-Mor

End Alg-Tun-Mor

End Alg-Tun-Mar

Ibero-maghrebian

Ibero-maghrebian

End Alg-Tun-Mor

End Alg-Tun-Mar

End Alg-Tun-Mor

$$
\text { Tarja }
$$

Sedrata

Ben Attia

Ben Attia

Majen Matlag

Mechrouha

Mare Ain Zena,

Oued Medjarda, Majen Matlag

Ben Attia

Sedrata

Mechrouha, Djebel Mcid

Ain Talhi

Sedrata

Sedrata

Ain Talhi,

Mechrouha

Hannecha,

Ain Talhi,

Hannecha, Ain senour

Hannecha, Ain senour

$$
\text { Tarja }
$$

Ain Talhi

Ain Talhi

Djebel Mcid,

Hannecha, Ain

$$
\text { Zena }
$$

Mazeraa

Hannecha

Hannecha, Ain

$$
\text { Zena }
$$

Majen Matlag

Ben Attia, Sedrata

Ain senour,

Tiffech, Ain

$$
\text { Zena }
$$

Mechrouha
Matorral

Humid

environment

Matorral

Lawn

Steppe

C

$\mathrm{R}$

$\mathrm{R}$

$\mathrm{R}^{*}$

FC

C

Cork oak forest $\quad$ C

Cork oak forest $\quad \mathrm{R}$

Matorral $\mathrm{R}$

Steppe $\quad$ FC

Steppe $\quad$ R

Steppe C

Humid

environment

Cork oak forest $\quad \mathrm{R}$

Humid

environment

Steppe

Steppe

Cork oak forest, matorral

Matorral

Steppe

Steppe

Matorral, cork oak forest

Matorral, cork oak forest

Matorral, lawn $\quad \mathrm{R}^{*}$

Lawn, matorral $\quad \mathrm{R}$

Matorral

R*

Matorral

VR*

Matorral

$\mathrm{VR}^{*}$

Matorral, cliff $\quad$ C

Zeen oak forest $\quad$ VR

Matorral

$\mathrm{R}$

Matorral, cliff

$\mathrm{R}$

Humid

environment

VR

Steppe

FC

Lawn, cliff

C 
Bulletin de la Société Royale des Sciences de Liège, Vol. : 90, articles, 2021, p. 213 - 240

Plagius maghrebinus Vogt \& Greuter

Asteraceae

Psychine stylosa Desf.

Ranunculus bulbosus subsp. aleae (Willk.) Rouy \& Foucaud

Reichardia tingitana subsp. discolor (Pomel) Jahand. \& Maire

Rhaponticum acaule (L.) DC.

Romulea ligustica Parl.

Rosmarinus eriocalyx Jord. \&

Fourr. subsp. eriocalyx

Rupicapnos numidica (Coss. \& Durieu) Pomel

Sambucus ebulus L.

Sambucus nigra L.

Santolina africana Jord. \& Fourr.

Scrophularia tenuipes Coss. \& Durieu ex Coss.

Scutellaria columnae All.

subsp. columnae

Sedum cepaea L.

Sedum pubescens Vahl

Serapias lingua L. subsp. lingua

Serapias stenopetala Maire \&

T. Stephenson

Sideritis incana L. subsp. incana

Sinapis pubescens subsp. indurata (Coss.) Batt.

Smyrnium perfoliatum $\mathrm{L}$.

Stachys duriaei de Noé

Teucrium atratum Pomel

Thymus algeriensis Boiss. \& Reut.

Thymus munbyanus subsp. coloratus (Boiss. \& Reut.) Greuter \& Burdet

Veronica montana $\mathrm{L}$.

Vinca major L.

Viola munbyana Boiss. \& Reut.

Viola riviniana Reich.

Brassicaceae
Ranunculaceae

Asteraceae

Asteraceae

Iridaceae

Lamiaceae

Papaveraceae

Adoxaceae

Adoxaceae

Asteraceae

Scrophulariaceae

Lamiaceae

Crassulaceae

Crassulaceae

Orchidaceae

Orchidaceae

Lamiaceae

Brassicaceae

Apiaceae

Lamiaceae

Lamiaceae

Lamiaceae

$\mathrm{Ch}$

Lamiaceae

Ch

Plantaginaceae

Apocynaceae

Violaceae

Violaceae

Th

Th

Hem

Geo

$\mathrm{Ch}$

$\mathrm{Ch}$

$\mathrm{Ch}$

Hem

Th

$\mathrm{Ch}$
End Alg-Tun-Mor
$\mathrm{Ch}$

End Alg-Tun-Mor

Th

Mediterranean

End Alg-Tun

End Alg-Tun

Eury-

Mediterranean

Eury-

Mediterranean

End Alg-Tun-Mor

Hem

End Alg-Tun

Mediterranean

Mediterranean-

Atlantic

End Alg-Tun

Mediterranean

End Alg-Tun

Mediterranean

End Alg

Eury-

Mediterranean

End Alg-Tun

End Alg-Tun$$
\text { Zen }
$$

Mazeraa

Mechrouha,

Oued Medjarda,

Majen Matlag

Ben Attia,

Sedrata

Majen Matlag

Zeen oak forest,

cork oak forest,

humid environment

Steppe

Humid

environment

Ben Attia

Steppe

Lawn

Ain senour

Tiffech

Ain Talhi, Mare

Ain Zena, Ain

Zena, Ouled

Bechih, Djebel

Mcid

Matorral, humid

environment, cliff,

cork oak forest

Hannecha

Ain Zena

Matorral

Cliff

Ain Trab, Oued

Medjarda

Oued Medjarda

Ben Attia,

Merahna,

M'daourouche

Humid

environment

Humid

environment

Steppe

Mazeraa

Zeen oak forest

Mazeraa

Mazeraa, Ouled

Bechih

Tarja

Ain Talhi, Mare Ain Zena

Mare Ain Zena

Ben Attia,

M'daourouche

Ain Zena,

Hannecha,

Djebel Mcid

Zeen oak forest

R

Zeen oak forest,

cork oak forest

R

Matorral FC

Humid

environment

Humid

environment

Steppe

Cliff, matorral

Mazeraa

Hannecha, Ain

Zena

Mechrouha

Hannecha, Ain

Zena, Ben Attia

Djebel Mcid,

M'daourouche,

Ain senour

Tiffech

Mecherouha,

Ouled Bechih,

Ain Zena

Hem

End Alg-Tun-Mor

Pool of Ain Zena

Ch Mediterranean

Mechrouha

Hem End Alg-Tun-Mor

Djebel Mcid

Mezeraa,

Mechrouha
Zeen oak forest $\quad \mathrm{R}$

Matorral, cliff $\quad$ FC

Cork oak forest $\quad \mathrm{R}$

Matorral, cliff, C

lawn, steppe

Cork oak forest,

$$
\text { cliff }
$$

Humid

environment

Cork oak forest

Matorral

Zeen oak forest,

Zeen oak forest,
cork oak forest

End: Endemic, Subend: Subendemic Alg: Algeria; Tun: Tunisia; Mor: Morocco; Lib: Libya; Egy: Egypt; Maur:

Mauritania; Itl: Italy; Spa: Spain; Bio. T: biological type; Th: Therophyte; Hem: Hemicryptophyte; Ch:

Chamaephyte; Geo: Geophyte; Ph: Phanerophyte; Hydr: Hydrophyte; C: common; F: fairly; R: rare; VR: very

rare; $(*)$ modified rarity. 
Bulletin de la Société Royale des Sciences de Liège, Vol. : 90, articles, 2021, p. 213 - 240

Of all the taxa studied, ten were threatened or near-threatened as registered on the IUCN Red List (IUCN, 2021), while 13 species were protected by Executive Decree No. 12-03 in Algeria (J.O.R.A, 2012) (see Table 3).

Table 3: List of species protected according to Executive Decree $n^{\circ} 12-03$ (JORA, 2012) and/ or evaluated according to IUCN (2021)

\begin{tabular}{|c|c|c|}
\hline Taxa & JORA (2012) & IUCN (2021) \\
\hline Argyrolobium saharae Pomel & $\overline{\mathrm{P}}$ & \\
\hline Bunium crassifolium (Batt.) Batt. & $\mathrm{P}$ & \\
\hline Cedrus atlantica (Endl.) Carrière ex Manetti & $\mathrm{P}$ & EN \\
\hline Convolvulus durandoi Pomel & $\mathrm{P}$ & NT \\
\hline Cyclamen africanum Boiss. \& Reut. & $\mathrm{P}$ & \\
\hline Dactylorhiza elata (Poir.) Soó & & NT \\
\hline Drimia anthericoides (Poir.) Véla \& de Bélair & & $\mathrm{EN}$ \\
\hline Illecebrum verticillatum $\mathrm{L}$. & $\mathrm{P}$ & \\
\hline Juncus heterophyllus Dufour & & NT \\
\hline Mandragora officinarum L. & $\mathrm{P}$ & \\
\hline Ononis aragonensis Asso & $\mathrm{P}$ & \\
\hline Orchis laeta Steinh. & & NT \\
\hline Orchis patens Desf. subsp. patens & $\mathrm{P}$ & \\
\hline Phlomis bovei de Noé & $\mathrm{P}$ & \\
\hline Pilularia minuta Durieu & & EN \\
\hline Pistacia atlantica Desf. & $\mathrm{P}$ & NT \\
\hline Scrophularia tenuipes Coss. \& Durieu ex Coss. & $\mathrm{P}$ & NT \\
\hline Serapias stenopetala Maire \& T. Stephenson & & CR \\
\hline Teucrium atratum Pomel & $\mathrm{P}$ & \\
\hline
\end{tabular}

P: protected, NT: near-threatened, EN: endangered, CR: critically endangered. 
Bulletin de la Société Royale des Sciences de Liège, Vol. : 90, articles, 2021, p. 213 - 240

\subsection{Biogeographic distribution}

We have identified three biogeographic sets in the studied flora:

\subsubsection{The Mediterranean set}

This set included 39 species (32.77\%) of the flora listed, 27 for the Mediterranean element (sensu stricto), eight for the Eury-Mediterranean connecting element, and four for the Atlantic-Mediterranean connecting element. In this set, the richest families were the Orchidaceae family, with seven taxa, and the Apiaceae and Lamiaceae, with four taxa for each.

\subsubsection{Nordic set}

This set was represented by three paleotemperate taxa (Hypericum androsaemum, Geranium dissectum and Geranium columbinum), one Eurasian taxon (Chaerophyllum temulum) and one Europaen taxon (Veronica montana L.).

\subsubsection{Endemic set}

This set was the most important group of the studied flora, with $75(63.02 \%)$ species. The existent 26 families were of endemic taxa, including the Asteraceae (11 endemics), followed by the Fabaceae and Brassicaceae (nine endemics each). The genus Ophrys was the most diverse with four taxa, followed by the genus Genista with three taxa.

These species belonged to eight sub-elements of endemism:

- Endemic to Algeria: Two taxa strictly endemic to Algeria were identified in our list (Drimia anthericoides and Sinapis pubescens subsp. indurata);

-Algerian-Tunisian endemics: The number of Algerian-Tunisian endemic taxa was the highest among all the groups, with 27 species or $36 \%$ of the endemic flora of the region. This number was noteworthy for a region situated at the Tunisian borders;

-Algerian-Moroccan endemics: This element was composed of two taxa (Cedrus atlantica and Centaurea involucrata);

- Endemic to Algeria, Tunisia and Italy: Six endemic taxa were noted in Algeria-Tunisia and extending to Italy (Biscutella raphanifolia, Jonopsidium albiflorum, Orchis patens subsp. 
Bulletin de la Société Royale des Sciences de Liège, Vol. : 90, articles, 2021, p. 213 - 240

patens, Euphorbia cuneifolia, Centaurea solstitialis subsp. schouwii, and Genista ferox subsp.ferox);

-Ibero-Maghrebian: This group had two species (Ononis aragonensis and Ophrys atlantica subsp. atlantica);

- Endemic to North Africa: The number of taxa in this group was appreciable, with 31 species or $41.33 \%$ of the endemic flora of the region. These taxa were often found in at least three countries of North Africa (Algeria, Tunisia, Morocco, and Libya) and sometimes extended to Egypt and Mauritania;

- Tyrrhenian sub-endemics: Here, three taxa were shared between Numidia, Kroumiria, and the Corso-Sardinian block and/or the Tyrrhenian island group;

- Betico-Maghrebian endemics (Algeria, Tunisia and Spain or Algeria, Morocco and Spain) were represented by a single taxon.

\subsection{Biological distribution}

According to the global list of recorded species, the composition of the biological spectrum showed that the hemicryptophytes, with their 38 taxa (31.93\%), were predominant over other life forms. Geophytes were fairly well represented with 30 species $(25.21 \%)$, followed by therophytes, chamaephytes and phanerophytes, with 20 (16.8\%), 17 (14.28 \%) and 10 (8.40 $\%$ ) species respectively. Hydrophytes were poorly represented with only four species (3.36 $\%)$.

The even biological distribution between species was also observed in the identified biogeographic elements, except for the four hydrophytic taxa which were typically Mediterranean.

\subsection{Distribution of rare and endemic species according to habitat types}

Matorrals, humid environments, and cork oak forests were the richest in rare species (more than 15 species each), while lawns were the poorest with only seven species.

Matorral showed most frequent endemism, including 31 taxa, followed closely by cliff ecosystems with 22 taxa. Zeen oak forests, steppes, and cork-oak forests were in an intermediate situation (respectively 14, 17, and 18 species). While, lawns and wetlands were the poorest in endemics (11 and seven species respectively).

From a bioclimatic point of view, rare and endemic taxa were found in the three bioclimatic stages of the study region, from humid to semi-arid. 
3.5 The influence of environmental and edaphic variables on the richness of rare and endemic plants
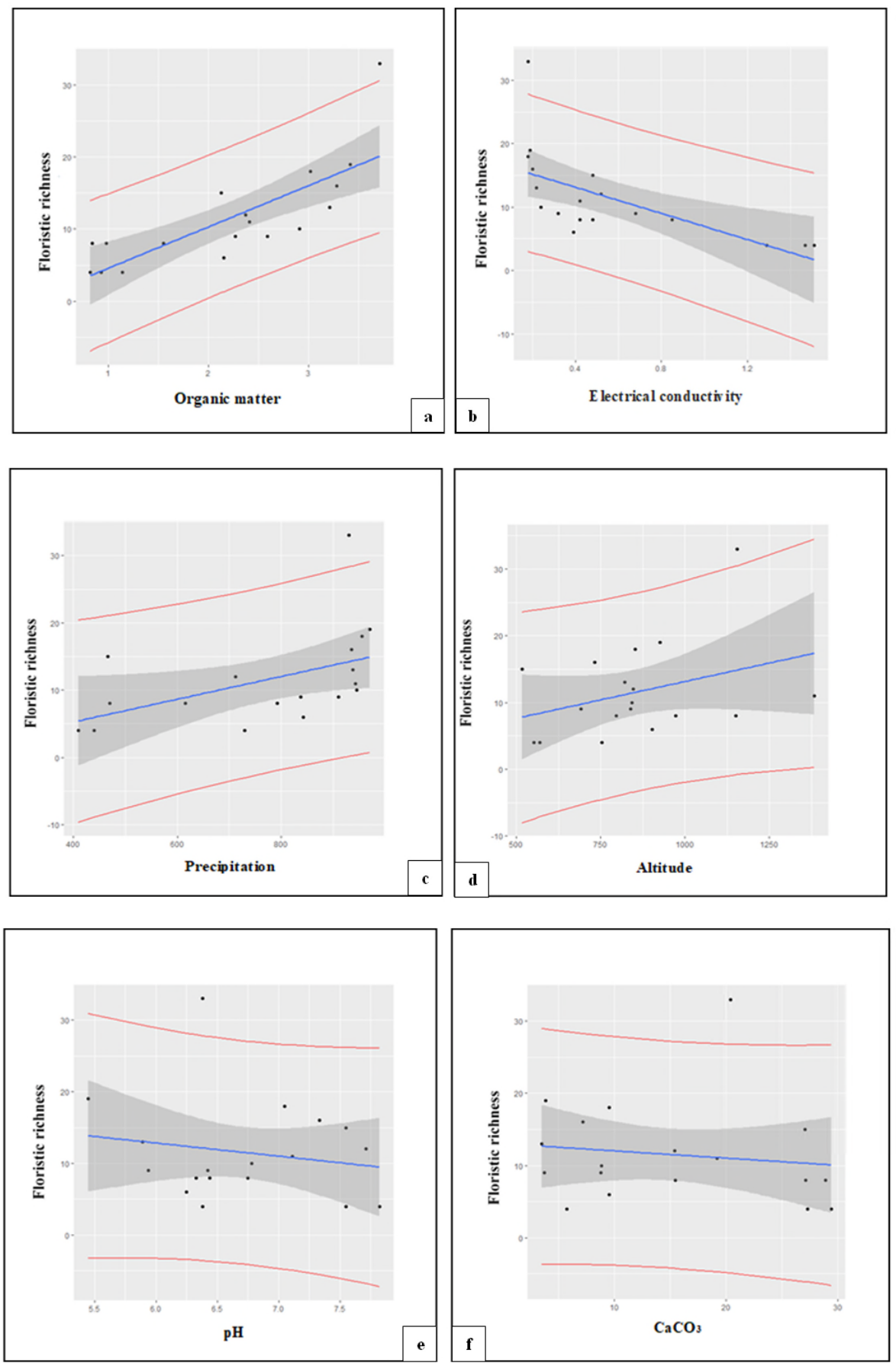

Figure 2: Correlations between the richness in rare and endemic species of the studied localities (black dots), (a) organic matter; (b) electrical conductivity; (c) precipitation; (d) altitude; (e) $\mathrm{pH}$; (f) total limestone 
Among all the studied sites, no significant regressions were observed between specific richness and topographic (altitude), climatic (precipitation), and edaphic variables $(\mathrm{pH}$, electrical conductivity, total limestone, and organic matter). Two variables were significant, namely organic matter $\left(R^{2}=0.6101 ; p=0.0001\right)$ and the electrical conductivity $\left(R^{2}=0.4103\right.$; $\mathrm{p}=0.0041$ ) (Fig. $2 \mathrm{a} \& \mathrm{~b}$ ). Other variables do not seem to influence the floristic composition hence the distribution of rare and endemic plants (Fig. 2 c, d, e \& f).

\section{Discussion}

\subsection{Characterization of the flora}

The rare and very rare flora of the study area had 119 taxa, i.e. $16.03 \%$ of the flora of the Souk Ahras region, based on 742 species according to the current state of our research (Hamel, Véla \& de Bélair, unpublished).

This first inventory of rare and endemic plants in the region of Souk Ahras highlighted the main aspects of this flora (changes in nomenclature, distribution, and type of endemism). Local researchers sometimes tend to neglect these aspects, however they should be considered as a priority in scientific research (Rao, 2004), towards the conservation of the local flora (Véla and Benhouhou, 2007). We observed some introduced individuals of Cedrus atlantica, heighting between 10 to $15 \mathrm{~m}$ and particularly present in the humid belt (the summit of Djebel Mcid, altitude $1380 \mathrm{~m}$ ), and its natural regeneration was ensured by the presence of small plants. The palynological work of Benslama et al. (2010) in the El Kala region (northeast of Algeria) hypothesizes the existence of cedar regional refugium in a relatively mild climate during the last glaciation, collateral to the hypotheses formulated by Terrab et al. (2006). Its presence would have benefited the glacial refugia, further east of its current range in Algeria and Tunisia, from which it disappeared following the contraction during the Holocene (Ben Tiba and Reille, 1982).

The large number of rare and endemic species recorded in the study region have been particularly identified in forest and pre-forest formations (matorrals, cork oak, and zeen oak forests). This richness in taxa accounts for the variability of biogeographical and ecological situations in connection with anthropogenic actions (Barbéro et al., 2001; Yahi et al., 2008).

Quézel (2000) confirmed the richness in endemic species or taxa, which grew on cliffs and rocks, with its disjunct area. Our observations, at an ecological level, concerning the site conditions involving rare and endemic species, were overall in agreement with Quézel and Santa (1962-1963). 
The observed plants were very unevenly distributed in the two biogeographical sectors (Quézel and Santa, 1962) of the study region. The Constantine sector (C1) had the highest number of rare and endemic plants (99 species), while the subsector of the highlands of Constantine (H2) was home to 27 high-value taxa. In fact, fluctuations in ecological conditions and the heterogeneity of habitats are determining factors of flora richness of these biogeographical zones (Touati et al., 2020).

Some species of $\mathrm{C} 1$ and $\mathrm{H} 2$ were not reported previously in "The flora of Algeria", e.g., Antirrhinum tortuosum, Argyrolobium saharae, Aristolochia paucinervis, Bunium crassifolium, Calamintha menthifolia, Castanea sativa, Chaerophyllum temulum, Daucus virgatus, Galactites mutabilis, Genista ulicina, Heliosciadium crassipes, Mandragora officinarum, Ononis angustissima subsp. polyclada, Ophrys atlantica subsp. hayekii, Orchis laeta, Orchis patens subsp. patens, Phlomis bovei, Pilularia minuta, Reichardia tingitana subsp. discolor, Scrophularia tenuipes, Smyrnium perfoliatum, Teucrium atratum, Veronica montana and Viola riviniana. All these new observations in the two biogeographical sectors of the region encouraged us to carry out a meticulous search for taxa, which might have escaped in previous investigations, as in the case of the 16 taxa illustrated by Edough Peninsula of Boulemtafes et al. (2018) and the study of Pteris vittata L. in February 2016 in Western Numidia (Hamel et al., 2020a). In a more global context, the presence of these plants in other biogeographical sectors suggested the requirement of a revision of the Algerian flora (cf. eflora Maghreb).

Other rare and endemic taxa, such as Euphorbia helioscopia subsp. helioscopioides (Loscos \& C. Pardo) Nyman and Odontites discolor Pomel, were found in our region. They seem to have previously disappeared or declined in the same habitat (Battandier and Trabut, 1890; Maire, 1928; Quézel and Santa, 1963). In some cases, natural environments have been so disrupted by other elements, such as fires, overgrazing, and urban development, that some sites were destroyed Considering the established uncertainity, additional surveys could be carried out in the future.

Mandragora officinarum, defined as rare and endangered in Quézel and Santa (1962), has been exclusive to the Kabylias-Numidia sector $(\mathrm{K})$ and the Algerian coastal sub-sector (A1) (site not found until now) (see Hanifi et al., 2007). We have observed it on the steppes of Ben Attia, which seems very rare and new for the subsector $\mathrm{H} 2$ of the Constantine highlands (Touati et al., 2020).

We have noticed that endemism status of some taxa had been changing, such as for Genista ferox subsp. ferox, previously mentioned as strictly endemic to the Maghreb by Quézel and 
Santa (1962) and recently retained as endemic in Algeria, Tunisia, Morocco, and Sardinia by CJB (2020). This is the case of Aristolochia paucinervis, previously mentioned in the flora of Quézel and Santa (1962-1963) as Mediterranean, while APD (2020) considers this as a Tyrrhenian subendemic. The changes in the chorological type of these species can be explained by the increased botanical investigations, either in the Mediterranean or in North Africa. Such investigations aim at listing biodiversity and monitor its evolution, either expanding the floristic lists by adding taxa or narrowing them by removing others (Gordo and Hadjadj-Aoul, 2019).

\subsection{Taxonomic, biological and biogeographic diversity}

A total of 119 remarkable taxa (endemic, rare, threatened, or protected) of angiosperms and pteridophytes were reported in our study sites. All these listed species are factors of a great conservation value, either for heritage reasons or for their risk of extinction (Pimm et al., 1988; Gaston et al., 1998).

The highest degree of endemicity corresponds to Asteraceae (11 taxa), Brassicaceae (nine taxa), Fabaceae (nine taxa), Orchidaceae (eight taxa) and Lamiaceae (seven taxa). These results corroborate the conclusions by Le Houérou (1995), who stated that the Asteraceae, Fabaceae, and Lamiaceae are the richest families in endemics in North Africa, and by Quézel (1978) regarding the Lamiaceae family. The richness of the local flora in Orchids in the study area confirmed the observations made by Boukehili et al. (2018). This resemblance is the result of their common history from which resulted a very homogeneous biogeographical unit (Quézel, 1964).

The relative number of endemic taxa sensu lato presented in this work was $63.05 \%$. However, it is comparably very high to that stated by Hamel et al. (2013) for the Edough peninsula (47\%), Djebbouri and Terras (2019) for the forest formations of Saïda (North-West Algeria) (08.81\%), Medjahdi et al. (2009) for the Traras mountains (19.71\%) and Miara et al. (2017) for the Tiaret region (38\%). This richness was the result of climate diversity, exposures, substrates, and orography with a mountain range, which culminates at $1405 \mathrm{~m}$ (Djebel Mcid), $1230 \mathrm{~m}$ (Saïda),1008m (Edough) and875m (Tarras). As stated by Verlaque et al. (1997), the Mediterranean endemism is mainly concentrated in mountain ranges and islands.

We have identified two restricted endemic species (endemic to Algeria). These taxa were both rare and endemic "taxa classified as of high heritage value", Drimia anthericoides was on the red list of the IUCN (Véla and de Bélair, 2017), as “endangered”. These are: 
- Drimia anthericoides: endemic to northeastern Algeria seemed to be a new species rank to the national flora (Véla et al., 2016). It is a critical taxon considered as a variety of Urginea maritima (L.) Baker (Maire, 1958; Quézel and Santa, 1962). Nevertheless, it differs from other aggregate species/varieties of Charybdis maritima (L.) Speta [=Urginea maritima] in terms of characters of flowers, fruits, bulbs, leaves, and by ploidy level (Véla et al., 2016). A review of the sites on the Tunisian side would still be beneficial in order to verify any past confusion. However, the observation site is on private land, thus potentially threatened by agriculture and/or grazing.

- Sinapis pubescens subsp. indurata: the diversity of the genus Sinapis in Algeria is remarkable with eight species and subspecies, of which two are endemics (S. pubescens subsp. aristidis and subsp. indurata) (Dobignard and Chatelain, 2011). This taxon has been previously reported in Souk Ahras in three different localities: Mont Mahrouf by H. de Perraudière (without specifying a date?) and by Reboud (without date?) in Sgao and Djebel Mcid (Maire, 1965). We have consulted all the plates of APD (2020) labeled "Sinapis pubescens subsp. indurata" of which three plates "MPU009015, MPU009016 and S-G-9038" collected by $H$. de Perraudière $(7 / 16 / 1861)$ related to our species.

The Algerian-Tunisian endemism represented the majority of endemic taxa recorded with 27 species. However, these border endemics correspond less to specialized areas with highly endemic species, than to the vast biogeographical zones, where endemic species are locally less rare and even abundant (Véla and Benhouhou, 2007; Hamel et al., 2013).

Our study area had 26 endemic species from Northwest Africa, with at least three countries (Algeria, Morocco, Tunisia and/or Libya).

The presence of Tyrrhenian endemics in the flora studied (three taxa) could explain the past terrestrial connections of the Algerian Tellian and Tunisian coast with Tyrrhenia (Quézel, 1964; Hamel and Boulemtafes, 2017; Hamel et al., 2020b). The importance of the Mediterranean (sensu lato) element of the rare flora in the study area (40 species, or $31.61 \%$ ) was in agreement with Quézel (1983), who found a greater number of Mediterranean-type species $(40.13 \%)$ in the North African flora.

Two plants were classified as flagship species in the Algerian (IPA), called "El Kala 2": Scrophularia tenuipes and Scutellaria columnae. These two plants, along with Convolvulus durandoi, Orchis laeta, Phlomis bovei, and Drimia anthericoides, are found in a restricted area (occurrence between 100 and 5,000 km²). Additionally, Dactylorhiza elata and Serapias 
stenopetala, two restricted endemic species were found in less than $100 \mathrm{~km}^{2}$ (Yahi et al., 2012).

Some plants, including the strict endemic Sinapis pubescens subsp. indurata, which are rare nationally but locally common, exist in the study area. In general, this rarity is due to the limited extent of the subhumid climate in Algeria.

The studied flora was dominated by hemicryptophytes (31.09\%), which prefer fairly stable environments and soil rich in organic matter (Kazi Tani et al., 2010). This suggested that the rainfall, the weakness of the light, and the pastures of the undergrowth favored the development of hemicryptophytes. Barbéro et al. (2001) reported that their abundance in Maghreb countries is due to the presence of organic matter and moisture. In addition, the dominance of hemicryptophytes has also been confirmed in the rare and endemic flora of the Edough Peninsula (Hamel et al., 2013). Geophytes were also well represented with 30 species. Their rate is relatively higher in a forest environment than in lawns and steppe areas where they tend to fade (Barbéro et al., 1981). Chamaephytes were represented by 20 species or $16.8 \%$ of the studied flora. They would be well adapted to the phenomenon of soil aridification as they can develop variants to drought (Floret et al., 1990). On the other hand, in certain regions, the encrusted soils are thin and the continuous calcareous crust which covers them does not allow any plant rooting (Boudjadja et al., 2010). Gamoun et al. (2011) claim that sandy soil is more productive due to increased water impermeability than limestone soil, which reduces water penetration.

\subsection{Threats and conservation measures}

Due to insufficient documentation and studies, 109 of the rare and endemic plants in our study area and elsewhere in Algeria have not been yet assessed, according to the criteria established by IUCN (2021). Protection is urgent to a total of 106 rare and endemic species. Without it, they are threatened with extinction, especially since they are not placed on the list of protected plant species in Algeria (JORA, 2012).

The rare and endemic flora of the Souk Ahras region is undergoing an alarming degradation. Additionally, anthropogenic activities, especially fires, overgrazing, and uncontrolled exploitation of species known for their therapeutic virtues (e.g. Origanum vulgare subsp. glandulosum, Thymus algeriensis, Rosmarinus eriocalyx subsp. eriocalyx, Mandragora officinarum, Thymus munbyanus subsp. coloratus, Deverra scoparia subsp. scoparia, and Santolina africana) stand as threats to this flora. Other taxa are heavily consumed (Allium 
porrum subsp. polyanthum, Bunium crassifolium, Echinops bovei, Rhaponticum acaule, and Romulea ligustica) or used as ornamentals (e.g. Hedera algeriensis, Sambucus nigra, Iris inguicularis, and Cyclamen africanum) (see Sakhraoui, 2021).

Based on our study we propose the main short-term conservation solutions below:

- Habitat protection, including populations of threatened species where conservation problems are most critical (Cedrus atlantica, Drimia anthericoides, Pilularia minuta, and Serapias stenopetala) by creating "micro-reserves", thus providing sustainable conservation and leading to a representative natural habitat (Laguna et al., 2004).

- Modeling the distribution of each species for its reintroduction into its natural habitat or into new suitable areas, according to the species distribution models.

- Revise and update the list of protected plant species in Algeria according to the criteria of endemism, rarity, and threats, as both rare and endemic species have high conservation value.

\section{Conclusion}

Our analysis based on the current knowledge of the rare and endemic flora of the wilaya of Souk Ahras revealed a significant specific richness (119 species), characterized by 75 endemic species and 77 rare species. This regional endemic flora is hardly known as a large area of the study region and remains very poorly explored. The entire area located at the Algerian-Tunisian border, the steppes of Macrochloa tenacissima (L.) Kunth, and Artemisia herba-alba Asso, located in the south of the region, deserve to be thoroughly explored. Likewise, the relationship between scarcity and endemism was remarkable. Half endemic taxa in a broad sense were rare and listed on the protected plant species in Algeria, while the majority (more than a hundred) are not protected. Hence, it is necessary to protect these species in accordance to endemicity and rarity.

\section{References}

AFNOR, 1999, Qualité des sols - vocabulaire. Partie 2. Termes et définitions relatifs à l'échantillonnage. Paris.

Allem, M., Hamel, T., Tahraoui, C., Boulemtafes, A. and Bouslama, Z., 2017, Diversité floristique des mares temporaires de la région d'Annaba (Nord-Est Algérien). International Journal of Environmental Studies, 75(3), 405-424.

Amirouche, R. and Misset, M. T., 2009, Flore spontanée de l'Algérie: différenciation écogéographique des espèces et polyploïdie. Cahier d'Agriculture, 18, 474-480.

Anderson, S., 1994, Area and endemism. The Quarterly Review of Biology, 69, 451-471. 
APD: African Plants Database, 2020, Geneve: Conservatoire et Jardin botaniques de la Ville de Genève; Pretoria (SA): South African National Biodiversity Institute. http:// www.ville-ge.ch/musinfo/bd/cjb/africa.

Barbéro, M., Loisel, R., Médail, F. and Quézel, P., 2001, Signification biogéographique et biodiversité des forêts du bassin méditerranéen. Bocconea, 13, 11-25.

Barbéro, M., Quézel, P. and Rivas-Martinez, S., 1981, Contribution à l'étude des groupements forestiers et pré-forestiers du Maroc. Phytoceonologia, 9, 311-412.

Battandier, J.A., 1888-1890, Flore de l'Algérie: dicotylédones. A. Jourdan (ed.), Alger.

Battandier, J.A. and Trabut, L., 1895, Flore d'Algérie, contenant la description de toutes les plantes signalées jusqu'à ce jour comme spontanées en Algérie et catalogue des plantes du Maroc: Monocotylédones. A. Jourdan (ed.), Alger.

Ben Tiba, B. and Reille, M., 1982, Recherches pollenanalytiques dans les montagnes de Kroumirie (Tunisie septentrionale) : Premiers résultats. Ecologia Mediterranea, Marseille, VII(4), p. 75-86.

Benhouhou, S., Yahi, N. and Véla, E., 2018, Algeria (chapter 3 "Key Biodiversity Areas (KBAs) for plants in the Mediterranean region"), UICN 53-60.

Benslama, M., Andrieu-Ponel, V., Guiter, F., Reille, M., de Beaulieu, J-L., Migliore, J. and Djamali, M., 2010, Pollen analysis from two littoral marshes (Bourdim and Garaat ElOuez) in the El-Kala wet complex (North-East Algeria). Lateglacial and Holocene history of Algerian vegetation. Comptes Rendus Biologies, 333(10), 744-754.

Blanca, G., Cabezudo, B., Cueto, M., Lopez, C. F. and Torres, C. M., 2009, Flora Vasculair de Andalucía Oriental, 1-4. - Seville.

Blondel, J., Aronson, J., Bodiou, J.Y., and Boeuf, G., 2010, The Mediterranean Region: Biological Diversity in Space and Time (Oxford: Oxford University Press).

Boudjadja, A., Kaddour, B.H. and Pauc, H., 2010, Increasing the value of surface water storage for protected wildlife, particularly the emblematic Cuvier's Gazelle, in Mergueb Reserve (M'sila, Algeria). Revue d'Ecologie la Terre et la Vie, 65(3), 243-253.

Boudy, P., 1955, Economie forestière nord-africaine. Tome IV, Description forestière de l'Algérie et de la Tunisie. Ed. Larose, Paris.

Boukehili, K., Boutabia, L., Telailia, S., Menaa, M., Tlidjane, A., Maazi, M.C., Chefrour, A., Saheb, M. and Véla, E., 2018, Les orchidées de la wilaya de Souk Ahras (Nord-est Algerien): inventaire, écologie, répartition et enjeux de conservation. Revue d'Ecologie (Terre et Vie), 73 (2), 167-179.

Boulemtafes, A., Hamel, T., de Bélair, G. and Véla, E. 2018, Nouvelles données sur la distribution et l'écologie de seize taxons végétaux du littoral de la péninsule de l'Edough (Nord-Est algérien). Bulletin de la Société linnéenne de Provence, 69, 59-76.

Braun-Blanquet, J., Roussine, N. and Nègre, R., 1952, Les groupements végétaux de la France méditerranéenne. Dir. Carte Group. Vég. Afr. Nord, CNRS.

Cañadas, E. M., Giuseppe, F., Peñas, J., Lorite, J., Mattana, E. and Bacchetta, G., 2014, Hotspots within hotspots: Endemic plant richness, environmental drivers, and implications for conservation. Biological Conservation, 170, 282-291.

Communication de l'Office National de la Météorologie (CONM), station: Souk Ahras, période (1990- 2020). 
de Bélair G., Véla, E. and Boussouak, R., 2005, Inventaire des orchidées de la Numidie (NE Algérie) sur vingt années. Journal Europaischer Orchideen, 37, 291-401.

Djebbouri, M. and Terras, M., 2019, Floristic diversity with particular reference to endemic, rare, or endangered flora in forest formations of Saida (Algeria). International journal of Environment Studies, 1, 1-8.

Dobignard, A. and Chatelain, C., 2010-2013, Index synonymique de la flore d'Afrique du Nord 5. [Eds des Conservatoire et jardin botaniques de Genève].

Domina, G., Bazan, G., Campisi, P. and Greuter, W., 2015, Taxonomy and conservation in higher plants and bryophytes in the Mediterranean area. Biodiversity Journal, 6, 197204.

Floret, C.H., Galan, M.J., Le Floc H., Orshan, G. and Romane, F., 1990, Growth forms and phenomorphology traits along an environmental gradient: tools for studying vegetation. Journal of Vegetation Science, 1, 71-80.

Gamoun, M., Tarhouni, M., Ouled Belgacem, A., Hanchi, B. and Neffati, M., 2010, Effects of grazing and trampling on primary production and soil surface in North African rangelands. Ekológia (Bratislava), 29(2), 219-226.

Gaston, K.J., 1991, How large is a species geographic range? Oikos, 61(3), 434-438.

Gaston, K. J., Blackburn, T. M. and Spicer, J. I., 1998, Rapoport's rule: time for an epitaph? Trends Ecology Evolution, 13, 70-74.

Ghrabi-Gammar, Z., Daoud-Bouattour, A., Ferchichi, H., Gammar, AM., Muller, SD., Rhazi, L. and Ben Saad-Limam, S., 2009, Flore vasculaire rare, endémique et menacée des zones humides de Tunisie. Revue d'Ecologie (Terre Vie), 64, 19-40.

Gordo, B. and Hadjadj-Aoul, S., 2019, L'endémisme floristique algéro-marocain dans les monts des Ksour (Naâma, Algérie). Flora Mediterranea, 29, 129-142.

Hamaidia H. et Berchi S. 2018, Etude systématique et écologique des Moustiques (Diptera: Culicidae) dans la région de Souk-Ahras (Algérie). Entomologie faunistique, 71, 13-27.

Hamel, T., Seridi, R., de Bélair, G., Slimani, A. R. and Babali, B., 2013, Flore vasculaire rare et endémique de la péninsule de l'Edough (Nord-Est algérien). Revue Synthèse des Sciences et de la Technologie, 26, 65-74.

Hamel, T. and Boulemtafes, A., 2017, Découverte d'une endémique tyrrhénienne Soleirolia soleirolii (Urticaceae) en Algérie (Afrique du Nord). Flora Mediteranea, 27, 185-193.

Hamel, T., de Bélair, G., Slimani, AR., Boutabia, L. and Telailia, S., 2020a, Nouvelle station de Pteris vittata L. (Pteridaceae) en Numidie (Algérie orientale). Acta Botanica Malacitana, 45, 1-3.

Hamel, T., Saci, A. and de Bélair, G., 2020b, Redécouverte d'un subendémique tyrrhénien, Tuberaria acuminata (Viv.) Grosser, en Numidie (Nord - Est algérien), Bull. Soc. linn. Provence, 71, 243-247.

Hanifi N., Kadik L. et Gittonneau G.G. 2007, Analyse de la végétation des dunes littorales de Zemmouri (Boumerdes, Algérie). Acta Bot. Gallica, 154, 235-249.

Huang, J., Huang, J., Liu, C., Zhang, J., Lu, X. and Ma, K., 2016, Diversity hotspots and conservation gaps for the Chinese endemic seed flora. Biological Conservation, 198, 104-112.

IUCN, 2021, IUCN Red List of Threatened Plants. Version 2021-1. http://www.iucnredlist.org 
J.O.R.A., 2012, Décret exécutif du 18 janvier 2012, complétant la liste des espèces végétales non cultivées et protégées. Journal officiel de la république algérienne. 3-12/12 du 18-012012. 12-38.

Kazi Tani, Ch., Le Bourgeois, T. and Munoz, F., 2010, Aspects floristiques des adventices du domaine phytogéographique oranais (Nord-Ouest algérien) et persistance d'espèces rares et endémiques. Flora Mediterranea, 20, 29-46.

Laguna, E., Deltoro, V.I., Pérez-Botella, J., Pérez-Rovira, P., Serra, L., Olivares, A. and Fabregat, C., 2004, The role of small reserves in plant conservation in a region of high diversity in eastern Spain. Biological Conservation, 119(3), 421-426.

Le Houérou, H.N., 1995, Considérations biogéographiques sur les steppes arides du Nord de l'Afrique. Sécheresse, 6, 167-182.

Maire R., 1928, Origine de la flore des montagnes de l'Afrique du Nord. Mém. Soc. Biogéog., 2, 187-194.

Maire, R., 1952-1987, Flore de l'Afrique du Nord (Maroc, Algérie, Tunisie, Tripolitaine, Cyrénaïque et Sahara). 16 vols, Lechevalier, Paris.

Mansouri, S., Miara M.D. and Hadjadj-Aoul, S., 2018, Etat des connaissances et conservation de flore endémique dans la région d'Oran (Algérie occidentale). Acta Botanica Malacitana, 43, 23-30.

Mathieu, C and Pieltain, F., 2003, Analyse chimique des sols. Ed. Tec et doc. Lavoisier, Paris, $292 \mathrm{p}$.

Médail, F. and Diadema, K., 2006, Biodiversité végétale méditerranéenne et anthropisation: approches macro et micro-régionales. Annales de Géographie, 651, 618-640.

Medjahdi, B., Ibn Tattou, M., Barkat, D. and Benabedli, K., 2009, La flore vasculaire des Monts des Traras. Acta Botanica Malacitana, 34, 57-75.

Miara, M.D., Ait Hammou, M., Rebbas, K. and Bendif, H., 2017, Flore endémique, rare et menacées de l'Atlas tellien occidental de Tiaret (Algérie). Acta Botanica Malacitana, 42 (2), 271-285.

Miara, M.D., Ait Hammou, M., Dahmani, W., Negadi, M. and Djellaoui, A., 2018, Nouvelles données sur la flore endémique du sous-secteur de l'Atlas tellien Oranais “O3" (Algérie occidentale). Acta Botanica Malacitana, 43, 63-69.

Mittermeier, R.A., Gil, P.R., Hoffmann, M., Pilgrim, J., Brooks, T., Mittermeier, C.G., Lamoreux, J. and Da Fonseca, G.A.B., 2004, Hotspots Revisited: Earth's Biologically Richest and Most Endangered Terrestrial Ecoregions. Chicago.

Morrone, J.J., 2018, The spectre of biogeographical regionalization. Journal of Biogeography, 45, 282-288,

Myers, N., Mittermeier, R.A., Mittermeier, C.G., da Fonseca, G.A.B. and Kent, J., 2000, Biodiversity hotspots for conservation priorities. Nature, 403, 853-858.

https://doi.org/10.1038/35002501

Myers, N., 2003, Biodiversity hotspots revisited. BioScience, 53(10), 916-917.

Pignatti, S., 1982, Flora d'Italia, 3 Vol. Bologna.

Pimm, S.L., Jones, H.L., and Diamond, J., 1988, On the risk of extinction. The American Naturalist, 132(6), 757-785.

Primack, R.B., Sarrazin, F. and Lecomte, J., 2012, Biologie de la conservation. Dunod, Paris. 
Quézel, P., 1964, L'endémisme dans la flore de l'Algérie. Comptes Rendus de la Société de biogéographie, 361, 137-149.

Quézel, P., 1978, Analysis of the flora of Mediterranean and Saharan Africa. Annals of the Missouri Botanical Garden, 65, 479-537.

Quézel, P., 1983, Flore et végétation de l'Afrique du Nord, leur signification en fonction de l'origine, de l'évolution et des migrations des flores et structures de végétation passées. Bothalia, 14, 411-416.

Quézel, P., 1995, La flore du bassin méditerranéen, origine, mise en place, endémisme. Ecologia Mediterranea, 22(1-2), 19-39.

Quézel, P., 1998, La végétation des mares transitoires à Isoetes en région méditerranéenne, intérêt patrimonial et conservation. Ecologia Mediterranea, 24, 111-117.

Quézel, P., 2000, Réflexions sur l'évolution de la flore et de la végétation au Maghreb méditerranéen. Ibis Press, Paris, 117 ppQuézel, P. and Santa, S., 1962-1963, Nouvelle flore de l'Algérie et des régions désertiques méridionales. CNRS (Ed). Paris, 2 vols.

R Development Core Team, 2013, R: A language and environment for statistical computing.

R Foundation for statistical computing, Vienna, Austria. [http://www.R-project.org.]

Rao, M.K., 2004, The importance of botanical nomenclature and synonymy in taxonomy and biodiversity M. K. Current Science, 87, 602-606.

Raunkiaer, C., 1934, The life forms of plants and statistical plant geography. Oxford University Press, London.

Riemann, H. and Ezcurra, E., 2005, Plant endemism and natural protected areas in the peninsula of Baja California, Mexico. Biological Conservation, 122, 141-150.

Sakhraoui, N., 2021. La flore horticole cultivée dans l'Est algérien : état des lieux et stratégies de gestion durable. Thèse de Doctorat en Physiologie Végétale. Thèse de Doctorat, Université Mohamed El Cherif Messaadia, Algérie.

Terrab, A., Paun, O., Talavera, S., Tremetsberger, K., Arista, M. and Stuessy, T.F., 2006, Genetic diversity and population structure in natural populations of Maroccan cedar (Cedrus atlantica; Pinaceae) determined with cpSSR markers. American Journal of Botany, 93(9), 1274-1280.

Touati, L., Hamel, T. and Meddad-Hamza, A., 2020, Sur la présence d'Atriplex canescens (Amaranthaceae) en Algérie: écologie, taxonomie et biogéographie. Flora Mediterranea, 30, 33-38.

Véla, E. and Benhouhou, S. 2007, Évaluation d'un nouveau point chaud de biodiversité végétale dans le bassin méditerranéen (Afrique du Nord). Comptes Rendus Biologies, 330, 589-605.

Véla, E., de Bélair, G., Rosato, M. and Rosselló, J., 2016, Taxonomic remarks on Scilla anthericoides Poir. (Asparagaceae, Scilloideae), a neglected species from Algeria. Phytotaxa, 288 (2), 154-160.

Véla, E. and de Bélair, G., 2017, Charybdis anthericoides. The IUCN Red List of Threatened Species 2017:e.T111272454A111273406. https://dx.doi.org/10.2305/IUCN.UK.2017

Verlaque, R., Médail, F., Quézel, P. and Babinot, J.F., 1997, Endémisme végétal et paléogéographie dans le bassin méditerranéen. Geobios, 21, 159-166.

Yahi, N., Djellouli, Y and de Foucault, B., 2008, Diversités floristique et biogéographique des cédraies d'Algérie. Acta Botanica Gallica, 155(3), 389-402. 
Walter, K.S. and Gillett, H.J. 1998, 1997 IUCN red list of threatened plants. Compiled by the World Conservation Monitoring Centre. IUCN - the World Conservation Union. Gland, Switzerland \& Cambridge, UK.

Yahi, N., Véla, E., Benhouhou, S., de Bélair, G. and Gharzouli, R., 2012, Identifying Important Plants Areas (Key Biodiversity Areas for Plants) in northern Algeria. Journal of Threatened Taxa, 4, 2453-2765.

Zedam, A., 2015, Etude de la flore endémique de la zone humide du Chott El Hodna, inventaire et préservation. Thèse de Doctorat en écologie, Université de Sétif, Algérie.

\section{Declaration of Competing Interest}

No potential conflict of interest was reported by the authors.

ORCID : https://orcid.org/0000-0002-9770-6805 
Bulletin de la Société Royale des Sciences de Liège, Vol. : 90, articles, 2021, p. 213 - 240

\section{Appendix A.}

Some rare and endemic species from the wilaya of Souk Ahras

(All the photos: Hamel T., except the photo A.5: de Bélair G.).
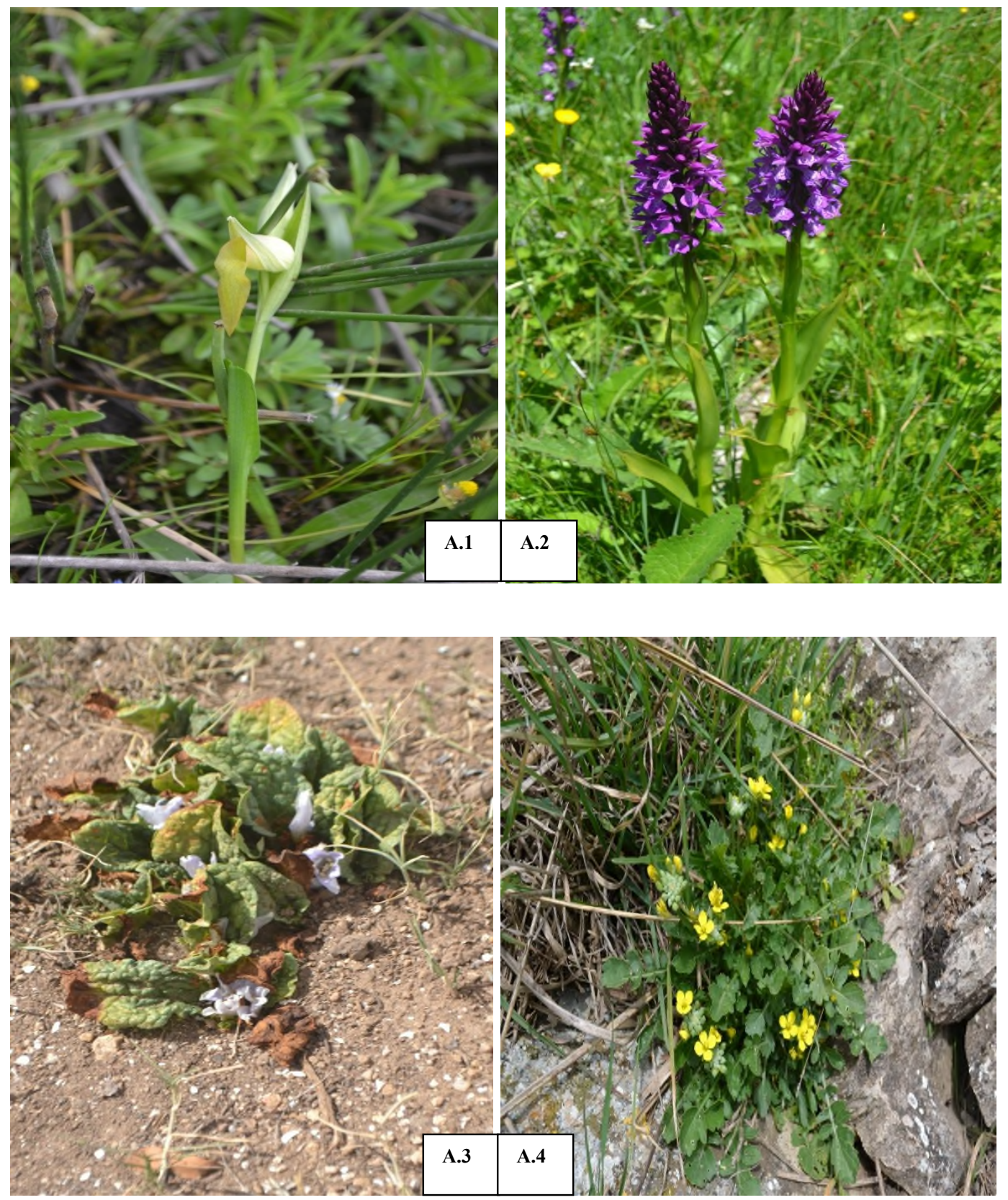
Bulletin de la Société Royale des Sciences de Liège, Vol. : 90, articles, 2021, p. 213 - 240
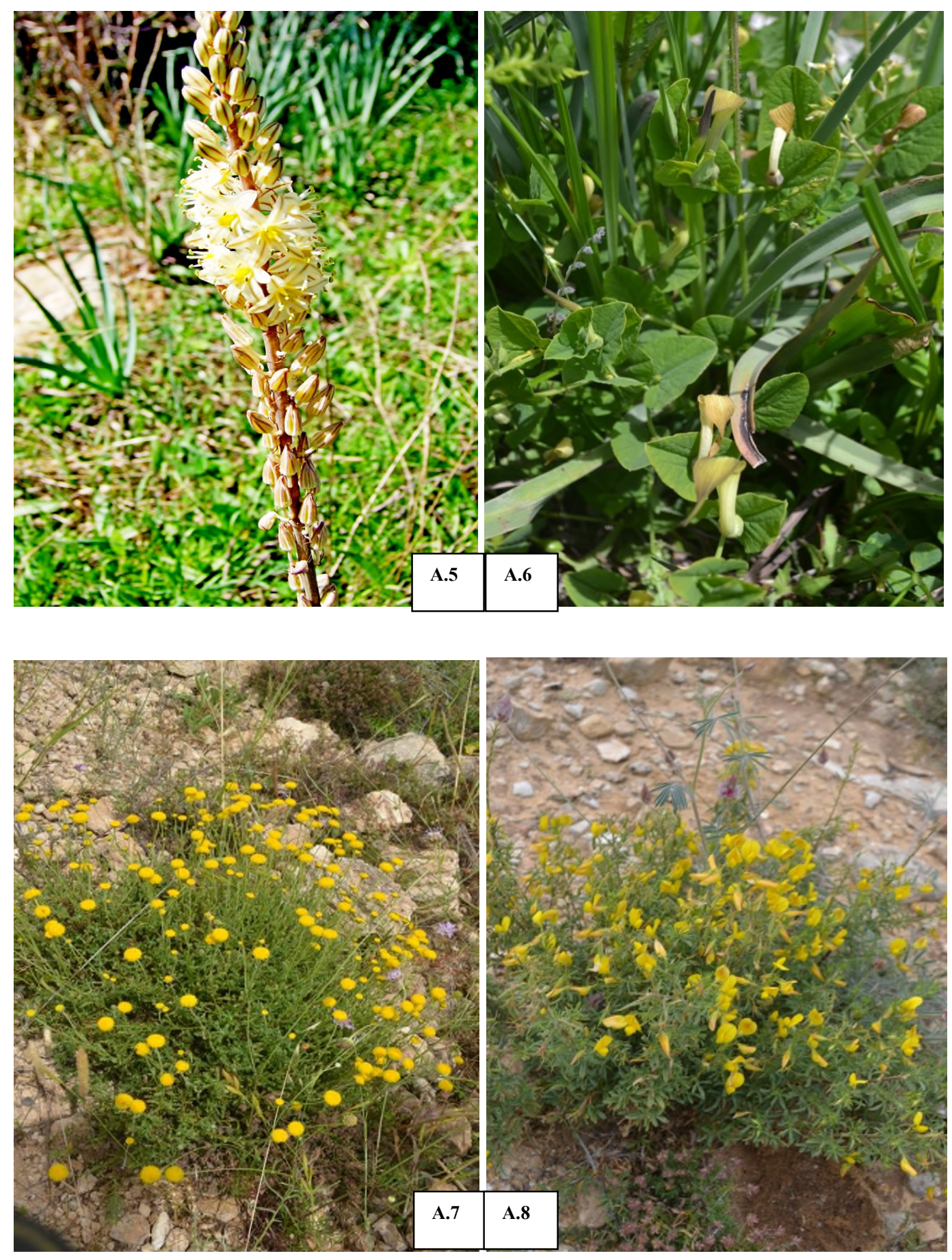
Bulletin de la Société Royale des Sciences de Liège, Vol. : 90, articles, 2021, p. 213 - 240
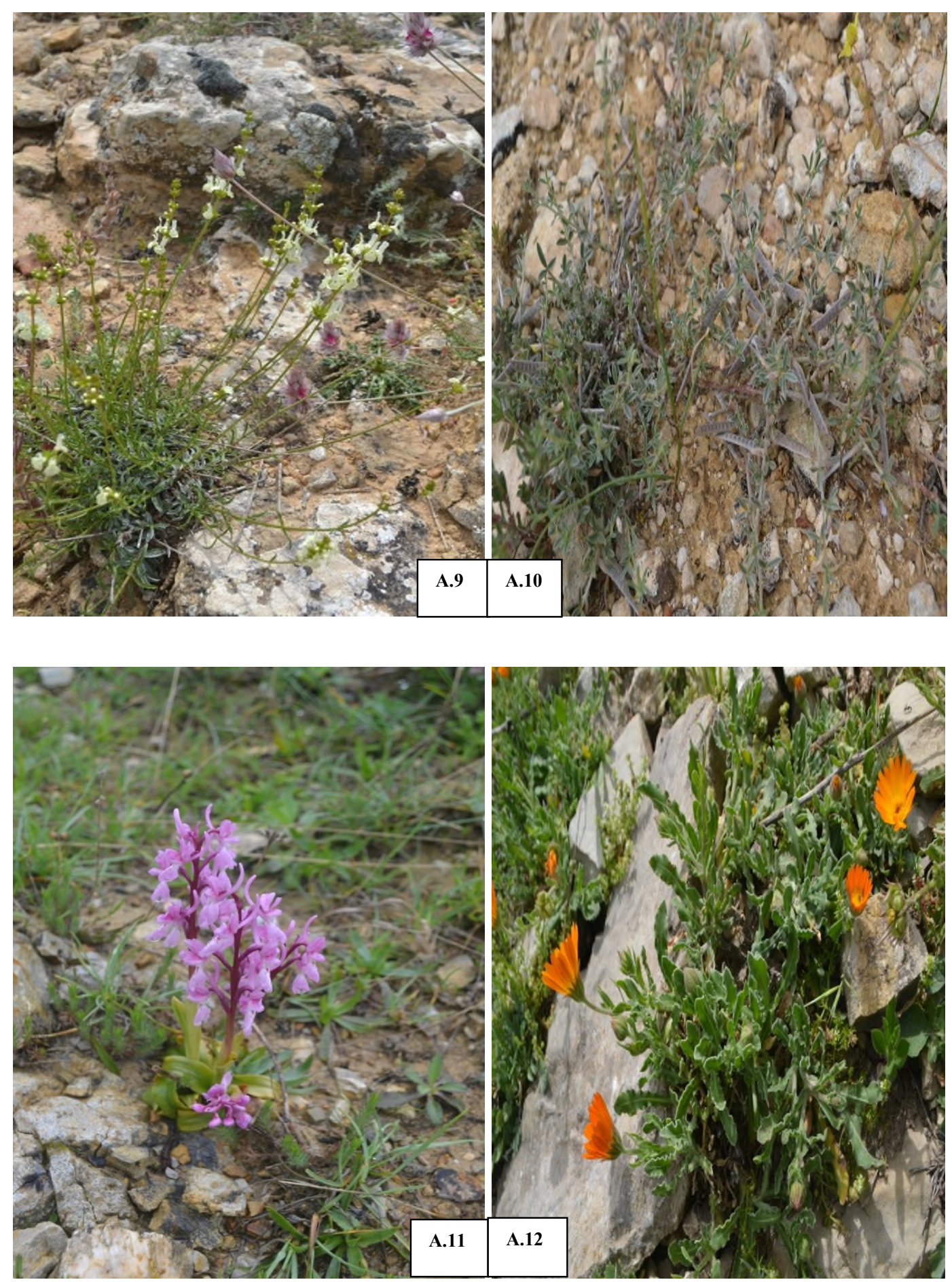
Bulletin de la Société Royale des Sciences de Liège, Vol. : 90, articles, 2021, p. 213 - 240
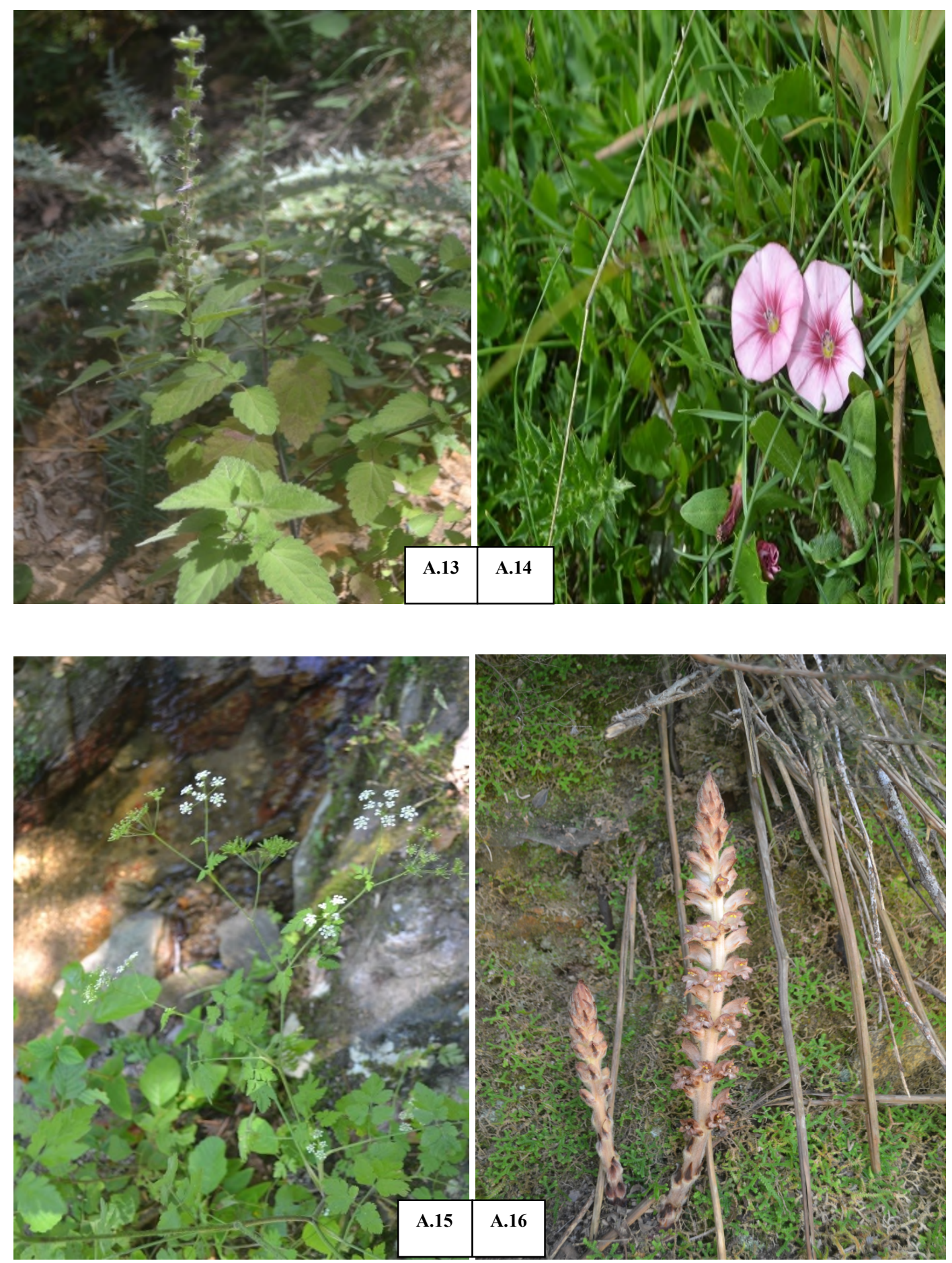

\begin{tabular}{|c|c|}
\hline A.1. Serapias stenopetala Maire \& T. Stephenson & A.9. Sideritis incana L. subsp. incana \\
\hline A.2. Dactylorhiza elata (Poir.) Soo & A.10. Argyrolobium saharae Pomel \\
\hline A.3. Mandragora officinarum $\mathrm{L}$. & A.11. Orchis laeta Steinh \\
\hline $\begin{array}{l}\text { A.4. Sinapis pubescens L. subsp. indurata (Coss.) } \\
\text { Batt }\end{array}$ & A.12. Calendula suffruticosa subsp. boissieri Lanza \\
\hline A.5. Drimia anthericoides (Poir.) Véla \& De Bélair & A.13. Scutellaria columnae All. subsp. columnae \\
\hline A.6. Aristolochia paucinervis Pomel. & A.14. Convolvulus durandoi Pome \\
\hline A.7. Santolina africana Jord. \& Fourr. & A.15. Chaerophyllum temulum L. \\
\hline A.8. Ononis angustissima subsp. polyclada Murb. & A.16. Orobanche rapum-genistae Thuill. \\
\hline
\end{tabular}

